\title{
Investigação de Marcadores Clínicos dos Estágios Iniciais da Doença de Alzheimer com Testes Neuropsicológicos Computadorizados
}

\author{
Helenice Charchat ${ }^{1}$ \\ Ricardo Nitrini \\ Paulo Caramelli \\ Koichi Sameshima \\ Universidade de São Paulo
}

\begin{abstract}
Resumo
O objetivo deste trabalho foi investigar marcadores clínicos dos estágios iniciais da Doença de Alzheimer (DA) utilizando testes neuropsicológicos computadorizados. Foram estudados 40 pacientes com diagnóstico clinicamente provável de DA (leves) e 73 idosos com envelhecimento normal. Os grupos foram pareados por sexo, idade e escolaridade. Os testes foram desenvolvidos com o programa MEL Professional e avaliaram memória episódica (ME), memória de curto-prazo (MCP) e tempo de reação de escolha (TRE). Em cada teste a porcentagem de acertos (PA) e a latência de resposta (LR) foram registrados. Ao comparar os grupos utilizando o teste t, o grupo DA apresentou PA significativamente menor que o grupo controle nos testes de ME e MCP e apresentou LR significativamente maior em todos os testes. A análise das curvas ROC mostrou que os testes ME, MCP e TRE foram sensíveis e específicos para discriminar os grupos e portanto foram os marcadores clínicos dos estágios iniciais da DA. Palavras-Chave: Testes computadorizados; neuropsicologia; doença de Alzheimer; diagnóstico; memória; tempo de reação.
\end{abstract}

Investigation of Clinic Markers of Early Alzheimer's Disease with Neuropsychological Computerized Tests

\begin{abstract}
The objective of this study is to investigate clinical markers of early Alzheimer's Disease (AD) using computerized neuropsychological tests. Forty patients with mild probable AD and 73 controls were studied. The groups were matched by sex, age and education. The MEL professional software was used to develop the tests. There were episodic memory (EM), shortterm memory (STM) and choice reaction time (CRT) tests. Percentage of correct responses (PCR) and response latency (RL) were registered on each test. The $t$ test was used to compare the groups. The AD group showed PCR significantly lower on EM and STM and showed RL higher on all other tests. The ROC analysis showed that EM, STM and CRT tests were sensitive and specific to discriminate the groups and therefore they were clinical markers of early AD.

Keywords: Computerized tests; neuropsychology; Alzheimer's disease; diagnosis; memory; reaction time.
\end{abstract}

O aumento significativo da população de idosos nos últimos anos em decorrência da elevação da expectativa de vida tem como principal conseqüência o aumento da prevalência das demências (IBGE, 1994; United Nations, 1986). As demências são síndromes caracterizadas por declínio de memória e outras alterações neuropsicológicas, que ocorrem preferencialmente no envelhecimento e com aumento exponencial em função da idade (Amaducci \& Lippi, 1990; Häfner, 1990). A doença de Alzheimer (DA) é a causa mais freqüente de demência e responde por

\footnotetext{
${ }^{1}$ Endereço para correspondência: Rua Conrado Niemeyer, 14, apto. 302, bloco A, 22021-050, Copacabana, Rio de Janeiro, RJ. E-mail: hcharchat@uol.com.br
}

cerca de $50 \%$ dos casos de demência em países ocidentais (Bachman e cols., 1993; Fratiglioni e cols., 1991; Herrera, Caramelli \& Nitrini, 1998). A DA se caracteriza pela presença de placas senis e emaranhados neurofibrilares nas regiões do hipocampo e córtex cerebral. Atualmente, o diagnóstico definitivo da doença só é possível mediante exame anátomo-patológico. Desta forma, a definição de critérios clínicos para o seu diagnóstico provável tornase fundamental.

Os critérios mais utilizados para diagnóstico da doença são os do NINCDS-ADRDA (National Institute of Neurological Comunicative Disorders and Stroke Alzheimer's Disease and Related Disorders) (McKhann e cols., 1984). Segundo estes critérios, o diagnóstico de demência deve ser estabelecido por uma avaliação clínica, 
documentado por exames de triagem e confirmado por testes neuropsicológicos. Os pacientes devem apresentar comprometimento progressivo em duas ou mais funções neuropsicológicas. Estas alterações devem prejudicar significativamente as atividades da vida diária. Desta forma, encontrar marcadores neuropsicológicos dos diferentes estágios da doença é importante para estudar a sua evolução clínica e auxiliar no diagnóstico.

O primeiro e mais evidente sintoma que emerge em pacientes com DA é o déficit de memória episódica anterógrada. Segundo Tulving (1995) memória episódica anterógrada refere-se à recordação de fatos e eventos recentes definidos no tempo e espaço. O comprometimento envolve a memória episódica e, num momento posterior, a memória semântica. Estas alterações mantêm paralelo com a evolução neuropatológica da doença (Caramelli e cols., 1998; Greene \& Hodges, 1996; Hodges \& Patterson, 1995). O déficit de memória episódica decorre de uma falha na aquisição e codificação de informações novas e a especificidade desta característica é mais saliente em testes de reconhecimento ou com auxílio de pistas semânticas (Christensen, Kopelman, Stanhope, Lorentz \& Owen, 1998; Mitrushina e cols., 1994).

Além de dificuldades de memória episódica associada ao sistema de longo-prazo, alguns autores como Morris e Baddeley (1988), Becker (1988) e Baddeley, Bressi, Della Sala, Logie e Spinnler $(1986,1991)$ descreveram déficit de memória de curto-prazo ou memória operacional em pacientes com DA. O conceito de memória operacional foi proposto por Baddeley e Hitch (1974) para entender e agrupar os numerosos experimentos sobre memória de curto-prazo. Eles sugeriram a substituição do conceito de estoque unitário da memória de curto-prazo pelo conceito de memória operacional constituído de subcomponentes. Eles identificaram três sub-componentes principais: centro executivo, alça fonológica e alça visuoespacial. O centro executivo é o sistema controlador da atenção que tem acesso à memória de longo-prazo e estabelece conexão com os dois sub-componentes subordinados (fonológico e visuo-espacial). Como controlador da atenção, ele seleciona os estímulos relevantes e coordena a análise dos estímulos de diferentes fontes e naturezas, sendo desta forma fundamental para executar qualquer tarefa cognitiva.

Morris e Baddeley (1988) mostraram que déficit no controle do centro executivo da memória operacional pode ser característico da DA, que explicaria o baixo desempenho em outras tarefas cognitivas. Esta hipótese está de acordo com os achados de Becker (1988) que apontou como os dois principais marcadores cognitivos da DA a dificuldade de aprendizagem explícita de informações novas e o déficit no controle do centro executivo da memória operacional.

Um outro parâmetro potencialmente útil para o diagnóstico e acompanhamento da evolução clínica da DA é o tempo de reação (TR) motor que permite medir o tempo de processamento das informações das várias etapas envolvidas em uma tarefa cognitiva pelo sistema nervoso central. Os testes com medida de tempo de reação são sensíveis para avaliar a integridade do sistema nervoso central (Müller, Richter, Weisbrod \& Klingberg, 1991; Nebes \& Brady, 1992). Assim, o registro do tempo de reação proporciona uma investigação mais refinada do funcionamento neuropsicológico do que a simples mensuração do número de respostas corretas e erradas. Autores como Kerr e colaboradores (1992), Nebes e Brady (1992) encontraram tempo de reação aumentado em pacientes nos estágios iniciais da DA. Apesar destas evidências, a mensuração do tempo de reação como marcador clínico tem sido pouco explorada na literatura.

O presente estudo tem como objetivo descrever marcadores neuropsicológicos dos estágios iniciais da DA utilizando como instrumento de avaliação uma Bateria de Testes Neuropsicológicos Computadorizados (BTNC) (Charchat, 1999). As principais vantagens de se utilizar testes computadorizados está justamente na possibilidade de, com precisão, controlar o tempo e a ordem de apresentação dos estímulos, registrar as latências e tipo de resposta e desenvolver várias formas de um mesmo teste. A BTNC foi desenvolvida como instrumental para avaliar quantitativamente funções neuropsicológicas acometidas e preservadas nos estágios iniciais da DA.

\section{Método}

\section{Participantes}

Foram estudados 40 pacientes com diagnóstico de DA provável definidos pelos critérios do NINCDSADRDA (McKhann e cols., 1984). Todos os pacientes foram considerados leves (CDR:1) pelos critérios do Clinical Dementia Rating (CDR; Hughes, Berg, Danzinger, Coben \& Martin, 1982). O CDR investiga a gravidade da demência ao contemplar avaliação de atividades básicas, instrumentais e cognitivas. O diagnóstico clínico dos pacientes baseou-se em: 1) exame neurológico, 2) exames laboratoriais, 3) avaliação neuropsicológica nãocomputadorizada, e 4) relato do cuidador ou neurologista da história de declínio cognitivo há pelo menos seis meses. Estes pacientes foram comparados a um grupo de 73 idosos com idade superior a 55 anos, com ausência de doenças neurológicas e psiquiátricas, sem queixa de 
declínio de memória e independentes para realizar as atividades da vida diária.

Os pacientes com DA e os controles não apresentaram diferenças significativas nas médias de idade, escolaridade e distribuição de freqüências de gêneros $(p>0,05)$. Os grupos apresentaram escores globais no Mini-Exame do Estado Mental (MEEM) estatisticamente diferentes (Tabela 1). Todos os participantes eram destros. No grupo dos pacientes com DA não foram incluídos analfabetos.

\section{Procedimento Geral}

Todos os sujeitos foram submetidos à BTNC. Seis testes neuropsicológicos foram desenvolvidos para avaliar memória episódica, memória de curto-prazo e tempo de reação, que estão descritos a seguir. A aplicação da bateria de testes computadorizados durava em média 40 minutos.

O procedimento geral para cada teste pode ser assim descrito:

Fase 1 - pré-teste: o sujeito executava uma tarefa breve como modelo. Esta fase tinha como finalidade a

Tabela 1. Características Demográficas e Clínicas dos Participantes

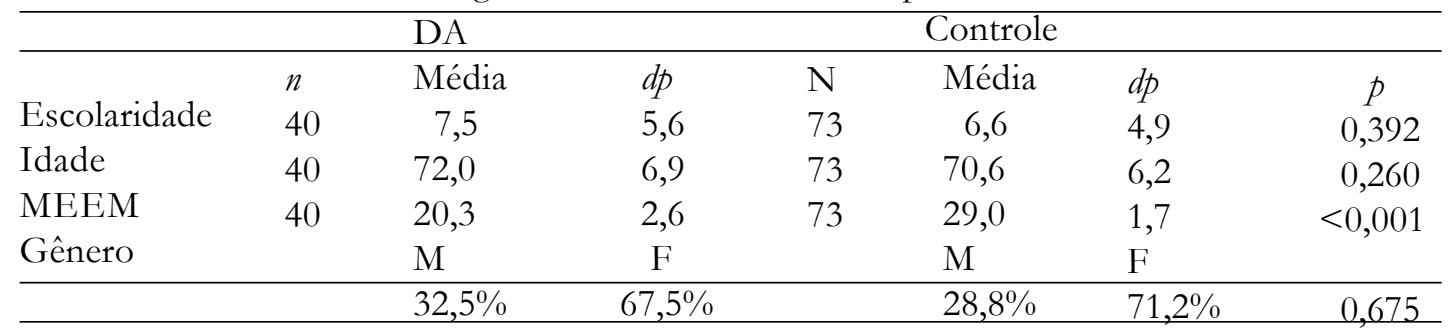

$\mathrm{N}$, número de participantes; $d p$, desvio-padrão da média; $p$, nível de significância pela utilização do teste $t$ Student; MEEM, Mini-Exame do Estado Mental; M, masculino; F, feminino.

\section{Aspectos Éticos}

Um consentimento informado era lido a todos os controles e/ou cuidadores dos pacientes que preenchiam os critérios de inclusão. Para participar da pesquisa eles deveriam assinar este documento. $\mathrm{O}$ consentimento informado, assim como todo o projeto da pesquisa, foi aprovado pelo comitê de ética em pesquisa do hospital onde os casos foram selecionados.

\section{Instrumentos}

Bateria de testes neuropsicológicos computadorizados (BTNC)

Os testes foram implementados com o programa MEL Professional versão 2.0 (Schneider, 1995) e administrados em um microcomputador IBM-PC compatível. Os estímulos foram apresentados em um monitor colorido SVGA com tela de 14 polegadas. A ordem e a apresentação dos estímulos foram controladas inteiramente pelos programas computacionais, bem como o registro do número e do tipo de respostas. A latência de resposta (tempo de reação - TR) foi mensurada com precisão de milissegundo. Uma caixa de resposta serial com cinco botões dispostos em linha e numerados de 1 a cinco foi utilizada como interface de entrada. A aplicação da bateria de testes computadorizados foi realizada em uma sala com atenuação acústica e iluminação parcialmente controlada. familiarização do sujeito com o computador e categoria de estímulos, a compreensão e memorização das instruções e procedimentos que foram utilizados durante o teste. Nesta fase, o desempenho do sujeito não foi registrado pelo computador. O examinador permanecia na sala junto ao sujeito.

Fase 2 - teste: executava o teste propriamente dito. Esta fase tinha a finalidade de avaliar uma determinada função neuropsicológica do sujeito. As instruções foram oferecidas ao sujeito oralmente pelo examinador e no momento seguinte, visualmente na tela do computador. A cada teste, as latências e o número de respostas corretas foram registradas automaticamente pelo computador. $\mathrm{O}$ examinador permanecia na sala junto ao sujeito para monitorar eventuais problemas de compreensão ou dificuldade de recordar as instruções.

Ao final de cada teste, o programa de computador selecionava aleatoriamente um novo conjunto de estímulos. Este recurso computacional de aleatorização permitiu desenvolver um grande número de variantes de um mesmo teste em que se pôde alterar a seqüência e/ou o subconjunto de estímulos utilizados.

\section{Procedimentos Específicos de cada Teste Teste Faces}

Este teste objetivou avaliar memória episódica de estímulos visuais complexos (faces de pessoas) por intermédio de um teste de reconhecimento. 
O procedimento deste teste pode ser descrito pelos seguintes passos:

1) Instrução oral do examinador: "Serão apresentadas faces de pessoas que você não conhece. Preste muita atenção!"

2) Instrução escrita na tela do computador: "Memorize as seguintes figuras".

3) Apareciam na tela do computador dez faces de pessoas desconhecidas dispostas conforme a Figura 1 e permaneciam nesta posição por um período de dez segundos.

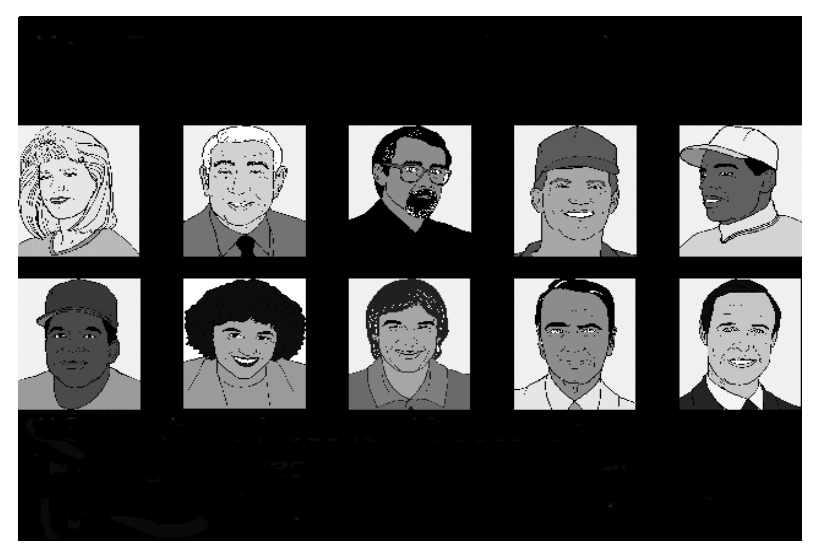

Figura 1. Categoria de estímulos do teste Faces

4) Instrução oral do examinador e visual escrita na tela do computador: "Agora serão mostradas novamente faces de pessoas que você não conhece, algumas que você já viu na primeira tela e outras novas. Quando a face já foi vista na tela anterior aperte o botão um (o examinador executava o movimento, apertando o botão na caixa de resposta), caso contrário aperte o botão três (o examinador executava o movimento, apertando o botão três na caixa de resposta).

5) Aparecia no centro da tela do computador uma face de pessoa desconhecida. Este passo foi executado 20 vezes, ou seja, foram apresentadas aleatoriamente e de forma individualizada as dez faces fixas expostas no passo três e dez faces distratoras que não foram expostas no passo três. Se o sujeito não pressionasse o botão um ou três até um tempo máximo de dez segundos, a próxima face seria apresentada.

6) Quando o sujeito pressionasse o botão um para as faces fixas e o botão três para as faces distratoras a resposta seria considerada correta. As latências destas respostas foram registradas

\section{Teste Desenhos}

Este teste objetivou avaliar memória episódica visual por intermédio de um teste de reconhecimento e aprendizagem de desenhos concretos.
O procedimento deste teste pode ser descrito pelos seguintes passos:

1) Instrução oral do examinador: "Será apresentada uma lista de desenhos. Preste muita atenção!"

2) Instrução escrita na tela do computador: "Memorize as seguintes figuras:"

3) Apareciam na tela do computador dez desenhos dispostos conforme a Figura 2 e permaneciam nesta posição por um período de dez segundos.

4) Instrução oral do examinador e visual escrita na tela do computador: "Agora você verá novamente desenhos, se o desenho fizer parte da lista que você viu na tela anterior aperte o botão um (o examinador executava o movimento, apertando o botão um na caixa de resposta), caso contrário aperte o botão três (o examinador executava o movimento, apertando o botão três na caixa de resposta).

5) Aparecia no centro da tela do computador um desenho. Este passo foi executado 20 vezes, ou seja, foram apresentadas aleatoriamente e de forma individualizada dez desenhos fixos expostos no passo três e dez desenhos distratores que não foram expostos no passo três. Se o sujeito não pressionasse o botão um ou três até um tempo máximo de dez segundos, o próximo desenho seria apresentado.

6) Quando o sujeito pressionasse o botão um para os desenhos fixos e o botão três para os desenhos distratores a resposta seria considerada correta. As latências destas respostas foram registradas.

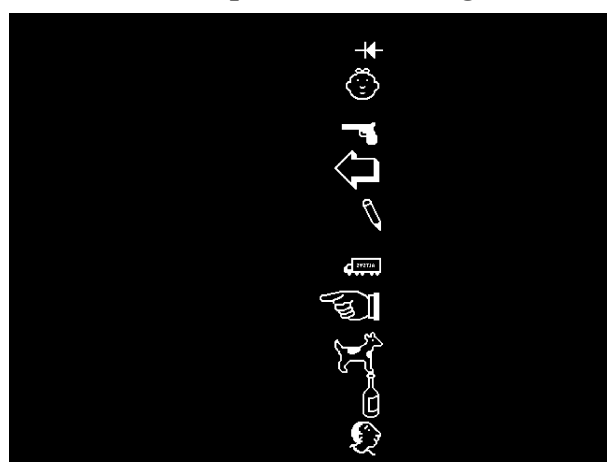

Figura 2. Categoria de estímulos do teste Desenhos

7) Os passos dois, três, cinco e seis foram repetidos três vezes para verificar efeito de aprendizagem da lista de desenhos fixa com a estratégia de repetição.

\section{Teste Palavras}

Este teste objetivou avaliar memória episódica de estímulos auditivo-verbais e visuais por intermédio de um teste de reconhecimento e aprendizagem de palavras.

O procedimento deste teste pode ser descrito pelos seguintes passos: 
1) Instrução oral do examinador: "Será apresentada uma lista de palavras, leia todas as palavras em voz alta" (Caso o sujeito apresentasse dificuldades de leitura o examinador poderia ler as palavras em voz alta)

2) Instrução escrita na tela do computador: "Memorize as seguintes palavras"

3) Apareciam na tela do computador dez palavras dispostas conforme a Figura 3 e permaneciam nesta posição por um período de dez segundos.

4) Instrução oral do examinador e visual escrita na tela do computador: "Agora você verá novamente palavras, se a palavra fizer parte da lista que você viu na tela anterior aperte o botão um (o examinador executava o movimento, apertando o botão um na caixa de resposta), caso contrário, aperte o botão três (o examinador executava o movimento, apertando o botão três na caixa de resposta).

5) Aparecia no centro da tela do computador uma palavra. Este passo foi executado 20 vezes, ou seja, foram apresentadas aleatoriamente e de forma individualizada as dez palavras fixas expostas no passo três e dez palavras distratoras que não foram expostos no passo três. Se o sujeito não pressionasse o botão um ou três até um tempo máximo de dez segundos, a próxima palavra seria apresentada.

6) Quando o sujeito pressionasse o botão um para as palavras fixas e o botão três para as palavras distratoras a resposta seria considerada correta. As latências destas respostas foram registradas.

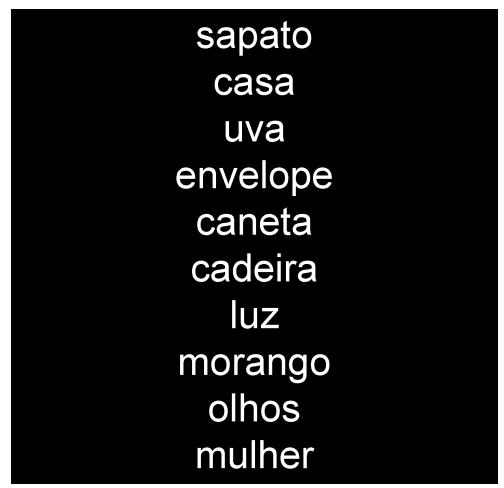

Figura 3. Categoria de estímulos do teste Palavras

7) Os passos dois, três, cinco e seis foram repetidos três vezes para verificar efeito de aprendizagem da lista de palavras fixas com a estratégia de repetição.

\section{Teste Formas Diretas}

Este teste objetivou avaliar atenção e percepção visual por intermédio de um teste de Tempo de Reação de escolha entre figuras geométricas iguais e diferentes.
O procedimento deste teste pode ser descrito pelos seguintes passos:

1) Instrução oral do examinador e escrita na tela do computador: "Serão apresentadas duas figuras. Se elas forem iguais aperte o botão um (o examinador executava o movimento, apertando o botão um na caixa de resposta), se elas forem diferentes aperte $\mathrm{o}$ botão três" (o examinador executava o movimento, apertando o botão três na caixa de resposta). "Seja o mais rápido que puder".

2) Apareciam no centro da tela do computador duas figuras geométricas (combinações de círculos, quadrados e triângulos). Este passo foi executado 50 vezes, ou seja, foram apresentadas aleatoriamente pares de figuras iguais e diferentes. Se o sujeito não pressionasse o botão um ou três até um tempo máximo de dez segundos, o próximo par de figuras seria apresentado.

3) Quando o sujeito pressionasse o botão um para os pares com figuras iguais e o botão três para os pares com figuras diferentes a resposta seria considerada correta. As latências destas respostas foram registradas. O Tempo de Reação neste teste mediu o tempo para o sujeito identificar dois estímulos, selecionar a resposta correta, selecionar o procedimento (botão um ou botão três) e executar o procedimento. A Figura 4 representa esquematicamente o teste.

Teste Formas Diretas
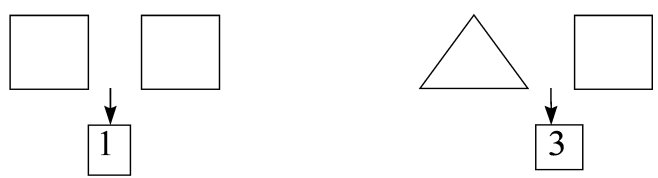

Teste Formas com Memória de Curto-Prazo

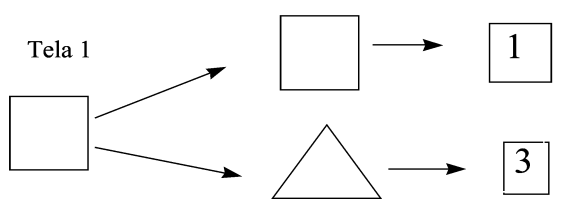

Figura 4. Categoria de estímulos e esquema do teste Formas Diretas e Formas com Memória de Curto-Prazo

Teste de Formas com Memória de Curto-Prazo

Este teste objetivou avaliar a memória de curto-prazo por intermédio de um teste de Tempo de Reação de escolha entre figuras geométricas iguais e diferentes separadas por intervalo de um segundo.

O procedimento deste teste pode ser descrito pelos seguintes passos:

1) Instrução oral do examinador: "Este teste será semelhante ao anterior. Só que ao invés de aparecerem 
as duas figuras ao mesmo tempo, aparecerá uma e depois a outra." (o teste anterior se refere ao teste Formas Diretas).

2) Instrução oral do examinador e escrita na tela do computador: "Será apresentada uma figura na tela. Preste atenção e memorize. Depois a tela apagará e aparecerá outra figura. Se as figuras forem iguais aperte o botão um (o examinador executava o movimento, apertando o botão um na caixa de resposta), se elas forem diferentes aperte o botão três" (o examinador executava o movimento, apertando o botão três na caixa de resposta). "Seja o mais rápido que puder".

3) Aparecia no centro da tela do computador uma figura geométrica (círculo ou quadrado ou triângulo) por um segundo.

4) A tela do computador apagava e permanecia assim por um segundo.

5) Aparecia no centro da tela do computador uma figura geométrica (círculo ou quadrado ou triângulo). A figura permanecia até o sujeito apertar o botão um ou o botão três na caixa de resposta. Se o sujeito não pressionasse o botão um ou três até um tempo máximo de dez segundos, uma nova figura aparecia na tela reiniciando o passo três.

6) Os passos três, quatro e cinco foram executados 50 vezes, ou seja, foram apresentadas aleatoriamente pares de figuras iguais e diferentes separadas por intervalo de um segundo.

7) Quando o sujeito pressionasse o botão um para os pares com figuras iguais e o botão três para os pares com figuras diferentes a resposta seria considerada correta. As latências destas respostas foram registradas.
O tempo de reação neste teste mediu a latência para o sujeito identificar o primeiro estímulo, identificar o segundo estímulo, recordar o primeiro estímulo e selecionar a resposta correta, selecionar o procedimento (botão um ou botão três) e executar o procedimento. A Figura 4 apresenta esquematicamente o teste.

\section{Teste Números}

Este teste objetivou avaliar a memória implícita por intermédio de um teste de Tempo de Reação serial utilizando dígitos como estímulos.

O procedimento deste teste pode ser descrito nos seguintes passos:

1) Instrução oral do examinador: "Será apresentado um número na tela. Aperte este número o mais rápido que puder na caixa de resposta. Se aparecer o número um, aperte o número um; se aparecer o número três, aperte o número três” (o examinador executava o movimento, apertando o botão correspondente na caixa de resposta).

2) Instrução escrita na tela do computador: "Será apresentado um número na tela, aperte este número o mais rápido que puder na caixa de resposta."

3) O sujeito escutava um tom e após $300 \mathrm{~ms}$ aparecia no centro da tela do computador um número (um ou três ou quatro ou seis).

4) O número permanecia até o sujeito apertar algum botão na caixa de resposta. Se nenhum botão fosse pressionado no tempo máximo de dez segundos, um novo número aparecia na tela reiniciando o passo três. 5) Os passos três e quatro foram executados 100 vezes.

Tabela 2. Funções Neuropsicológicas Avaliadas pelos Testes Computadorizados

Testes computadorizados Funções neuropsicológicas

Teste Faces

Teste Palavras

Teste Desenhos

Teste Formas Diretas

Teste Formas com Memória de Curto-Prazo

Teste Números

Diferença entre o teste Formas Diretas e o teste

Formas com Memória de Curto-Prazo

Diferença entre o teste de reconhecimento e aprendizagem de Palavras e Desenhos memória episódica anterógrada

atenção, percepção visual e tempo de processamento das informações

memória de curto-prazo

assimetria entre memórias verbal e visual 
6) Quando o sujeito pressionasse o botão correspondente ao número que estivesse aparecendo na tela do computador a resposta seria considerada correta. A latência destas respostas foram registradas.

A Tabela 2 mostra as funções neuropsicológicas avaliadas pela BTNC e quais testes correspondem a cada função. A seguinte seqüência de testes da bateria foi utilizada na avaliação dos sujeitos na BTNC: 1) Teste Formas Diretas; 2) Teste Formas com Memória de CurtoPrazo; 3) Teste Faces; 4) Teste Palavras; 5) Teste Números; 6) Teste Desenhos. Outros detalhes dos procedimentos da BTNC estão descritos em Charchat (1999).

\section{Análise Estatística}

Para uma padronização dos resultados dos testes, o número total de acertos em cada teste foi transformado em porcentagem de acertos e a média da latência de resposta medida em milissegundos (tempo de reação TR) foi transformada em logaritmo na base dez para aproximação da distribuição da normal e conseqüente utilização do teste $t$.

Análises com o uso de teste $t$-pareado foram desenvolvidas para avaliar a validade da diferença entre os testes Formas Diretas e Formas com Memória de Curto-Prazo como medida de memória de curto-prazo e da diferença entre o teste Palavras e Desenhos como uma forma de avaliar assimetria entre memória verbal e visual. $\mathrm{O}$ teste $t$-pareado comparou as médias de duas variáveis para um único grupo. As médias dos grupos (DA e Controles) em cada variável foram comparadas utilizando o teste t para amostras independentes com nível de significância de 0,01 .

Como primeira aproximação, o poder discriminativo de cada variável como possível marcador neuropsicológico dos estágios iniciais da DA foi verificado pela análise das curvas ROC (Receiver Operating Curves). Estas curvas são diagramas lineares que representam a relação entre positivos verdadeiros (sensibilidade) e falsos positivos (inverso da especificidade) para um conjunto de valores de corte. A análise da área sob a curva possibilita a avaliação e comparação da sensibilidade e especificidade de diferentes medidas ou testes em uma amostra. Cada curva representa o desempenho de uma variável, quando a curva se aproxima da reta diagonal, não há possibilidade de discriminação entre os grupos. Neste caso, a área sob a curva é 0,5. Quanto maior for esta área ou mais afastada da diagonal estiver a curva, melhor o poder discriminativo da variável. A seleção das variáveis com melhor capacidade de discriminar os grupos foi realizada pela comparação das áreas sob as curvas ROC.
Os cálculos estatísticos foram realizados com o uso do Programa SPSS (Statistical Package for the Social Science) e as curvas ROC foram desenvolvidas utilizando o aplicativo Excel (Microsoft) e o programa estatístico MedCalc.

\section{Resultados}

O teste $t$ de amostras independentes foi utilizado para comparar o desempenho do grupo dos pacientes com DA com o do grupo controle nos diferentes testes neuropsicológicos computadorizados. $\mathrm{O}$ teste $t$-pareado foi utilizado para analisar diferenças de desempenho entre os testes dentro do mesmo grupo.

\section{Memória episódica}

O grupo dos pacientes com DA apresentou tempo de reação médio significativamente maior que o grupo controle nos testes Faces $(p<0,01)$, Palavras $(p<0,01)$ e Desenhos $(p<0,01)$ (vide Figura 5).

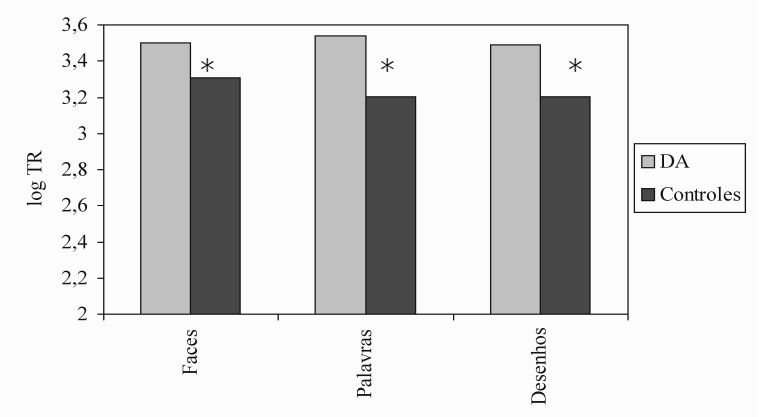

Figura 5. Comparação dos TR médios entre os grupos nos testes de memória episódica

O grupo dos pacientes com DA apresentou uma porcentagem de acertos significativamente menor que o grupo dos controles nos testes Faces $(p<0,01)$, Palavras $(p<0,01)$ e Desenhos $(p<0,01)$. A Figura 6 representa graficamente estes resultados.

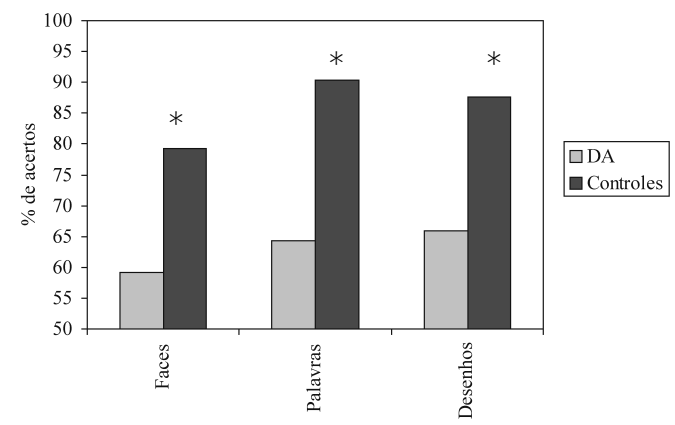

Figura 6. Comparação das médias de acertos entre os grupos nos testes de memória episódica Houve diferença significativa $(p<0,01)$ nos três testes $(*)$. 


\section{Assimetria entre Memória Verbal e Visual}

O desempenho do grupo com DA no teste Palavras (verbal) e no teste Desenhos (visual) foi comparado para verificar a existência de assimetria entre aprendizagem de estímulos verbais e visuais. O grupo com DA apresentou porcentagens de acertos médio de $64,4 \%$ no teste Palavras e $66 \%$ no teste Desenhos. Comparando estas porcentagens de acertos pelo teste t-pareado não foi possível observar diferença significativa intragrupo $(p>0,05)$. Em relação à latência de resposta também não foi evidenciada diferença significativa entre as tarefas $(p>0,05)$. Realizando a mesma análise para o grupo controle, eles apresentaram porcentagem de acertos médio no teste Palavras significativamente maior que no teste Desenhos $(p<0,05)$. Não houve diferença significativa entre os tempos de reação médios dos testes Palavras e Desenhos no grupo controle $(p>0,05)$. Além disso, ao comparar os três testes, a diferença no desempenho do teste Palavras, tanto para as medidas de tempo de reação como porcentagem de acertos, foi mais significante, ou seja, mostrou maior diferença entre os grupos $(p<0,05)$.

\section{Tempo de Reação de Escolha}

O grupo de pacientes com DA apresentou tempo de reação médio significativamente maior que o grupo controle nos testes de tempo de reação de escolha: teste Formas Diretas $(p<0,01)$, teste Formas com Memória de Curto-Prazo $(p<0,01)$ e teste Números $(p<0,01)$. A Figura 7 representa graficamente estes resultados.

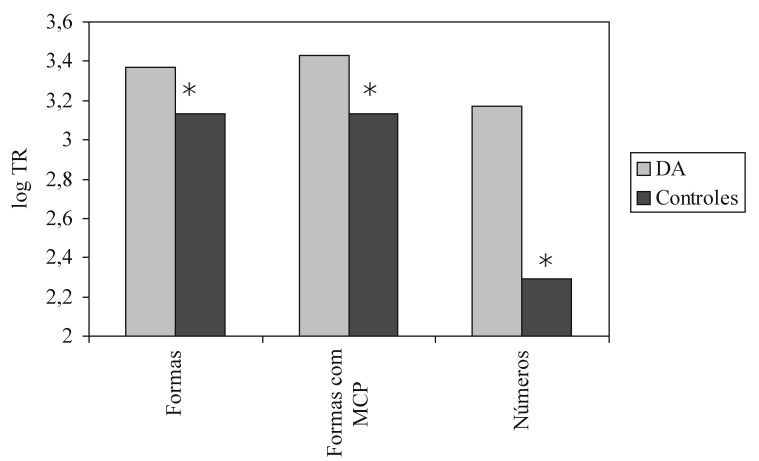

Figura 7. Comparações dos tempos de reação médios entre os grupos nos testes de tempo de reação de escolha. Houve diferenças significativas $(p<0,01)$ nos três testes $\left(^{*}\right)$.

O grupo dos pacientes com DA apresentou uma porcentagem de acertos significativamente menor que o grupo controle apenas no teste Formas com Memória de Curto-Prazo $(p<0,01)$. No teste Números e no teste Formas Diretas não foram observadas diferenças significativas entre os grupos $(p>0,05)$. A Figura 8 sumariza estes resultados.

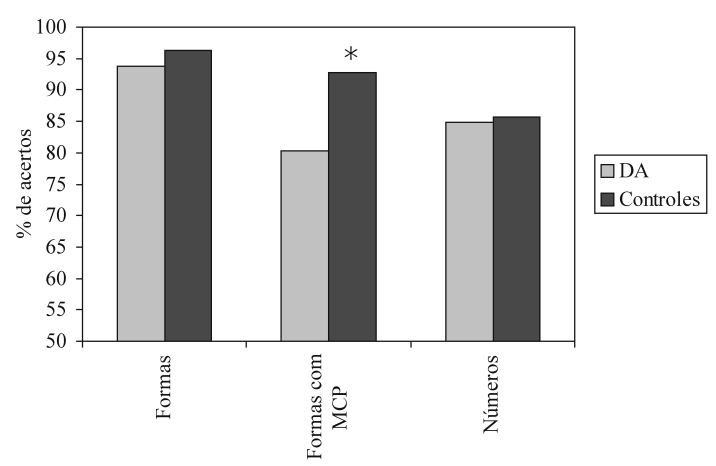

Figura 8. Comparações das médias de acertos entre os grupos nos testes de TR de escolha. Constatou-se diferença significativa $(p<0,05)$ entre os grupos DA e controles somente no teste Formas com Memória de Curto Prazo.

\section{Memória de Curto-Prazo}

Com a finalidade de analisar o efeito de uma complexidade adicional no teste Formas Diretas, elaborou-se uma variante com inclusão de um procedimento de memória de curto-prazo, denominado teste Formas com Memória de Curto-Prazo. O desempenho do grupo dos pacientes com DA nestes testes, Formas Diretas e Formas com Memória de CurtoPrazo, foi comparado. Os pacientes apresentaram a média da porcentagem de acertos no teste Formas Diretas $(93,8 \%)$ estatisticamente maior que a média da porcentagem de acertos no teste Formas com Memória de Curto-Prazo (80,3\%) $(p<0,01)$. Além disso, o grupo de pacientes com DA apresentou tempo de reação médio significativamente menor no teste Formas Diretas do que no teste Formas com Memória de Curto-Prazo $(p<0,05)$.

O grupo controle também apresentou a média da porcentagem de acertos no teste Formas Diretas significativamente maior que no teste Formas com Memória de Curto-Prazo $(p<0,01)$, contudo o tempo de reação médio nas duas tarefas foi semelhante $(p>0,05)$.

Apesar de os dois grupos apresentarem maior porcentagem de acertos do teste Formas Diretas quando comparado com o teste Formas com Memória de CurtoPrazo, o grupo com DA apresentou diferença entre as médias de 13,50, enquanto o grupo controle uma diferença de apenas 3,49.

\section{Marcadores Neuropsicológicos dos Estágios Iniciais da DA}

Para cada variável obtida na BTNC sua respectiva curva ROC foi traçada, a partir do que se calculou a área 
abaixo da curva, seu respectivo erro padrão, intervalo de confiança, sensibilidade e especificidade. A Tabela 3 mostra estes valores.

Tabela 3. Curvas ROC de cada Variável

\begin{tabular}{llllll}
\hline Variáveis & $\begin{array}{l}\text { Sensib. } \\
(\%)\end{array}$ & $\begin{array}{l}\text { Espec. } \\
(\%)\end{array}$ & AUC & EP & IC \\
\hline \% Palavras & 93,8 & 91,2 & 0,964 & 0,017 & $0,907-0,991$ \\
Log Tr Palavras & 82,8 & 89,1 & 0,932 & 0,034 & $0,860-0,974$ \\
\% Faces & 84,4 & 80,9 & 0,898 & 0,030 & $0,822-0,950$ \\
Log Tr Números & 82,8 & 85,9 & 0,867 & 0,046 & $0,781-0,929$ \\
Log Tr Formas Com Mcp & 75,9 & 92,2 & 0,866 & 0,046 & $0,779-0,928$ \\
\% Desenhos & 81,3 & 83,8 & 0,852 & 0,037 & $0,768-0,915$ \\
Log Tr Desenhos & 72,4 & 87,5 & 0,808 & 0,052 & $0,725-0,891$ \\
\% Formas Com Mcp & 62,5 & 85,3 & 0,817 & 0,042 & $0,727-0,887$ \\
Log Tr Faces & 72,4 & 73,0 & 0,808 & 0,053 & $0,713-0,883$ \\
Log Tr Formas & 62,1 & 85,9 & 0,800 & 0,056 & $0,685-0,861$ \\
\% Formas & 68,7 & 60,3 & 0,673 & 0,055 & $0,572-0,764$ \\
\% Números & 81,3 & 44,1 & 0,583 & 0,060 & $0,480-0,681$ \\
\hline
\end{tabular}

Legenda: Sensib., sensibilidade; Espec., especificidade; AUC, área sob à curva ROC; EP, erro padrão; IC, intervalo de confiança.

A análise das curvas ROC mostrou que as variáveis que avaliaram memória episódica e Tempo de Reação de escolha apresentaram maior capacidade de discriminar o grupo com DA do grupo controle. Estas variáveis apresentaram área sob a curva acima de 0,85 , ou seja, classificaram corretamente pelo menos $85 \%$ dos sujeitos. Conforme a Tabela 3, as variáveis com maior capacidade discriminativa foram: 1) Porcentagem de acertos do teste Palavras; 2) Tempo de reação médio do teste Palavras; 3) Porcentagem de acertos do teste Faces; 4) Porcentagem de acertos do teste Desenhos; 5) Tempo de reação médio do teste Formas com Memória de Curto-Prazo; 6) Tempo de reação médio do teste Números. As curvas ROC dessas variáveis mais discriminantes estão representadas na Figura 9.
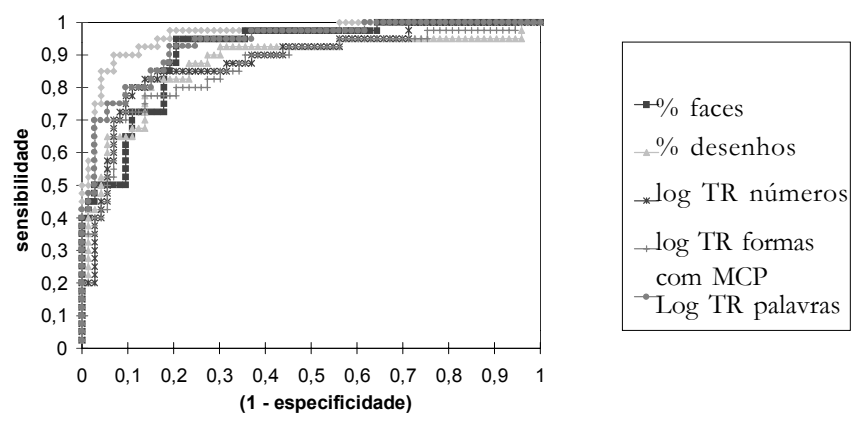

Figura 9. Curvas ROC das variáveis selecionadas investigação das características neuropsicológicas dos estágios iniciais da DA é fundamental para uma melhor compreensão da doença e o estabelecimento do seu diagnóstico precoce. Este estudo, ao comparar o desempenho nos diferentes testes da BTNC intragrupo e intergrupos, identificou alterações neuropsicológicas presentes nos estágios iniciais da DA. A BTNC incluiu testes de tempo de reação de escolha para avaliar atenção, percepção visual, memória de curto-prazo e velocidade de processamento das informações; além de testes de reconhecimento e aprendizagem de informações novas para avaliar memória episódica e assimetria entre memória verbal e visual.

\section{Memória Episódica}

Os estímulos de cada teste de reconhecimento são entidades únicas apresentadas em espaço e tempo definidos, sendo considerados parte do sistema de memória explícita, mais especificamente de memória episódica. O comprometimento acentuado do grupo com DA nos testes de memória episódica foi evidenciado por porcentagem de acertos diminuída e tempo de reação aumentado nos três testes aplicados.

A análise das curvas ROC reforçou estes resultados ao mostrar que os testes de memória episódica classificaram corretamente $85 \%$ ou mais dos sujeitos, a variável com maior capacidade discriminativa foi a porcentagem de acertos do teste Palavras (96\%) seguido pelo seu tempo de reação médio (93\%). 
Mitrushina e colaboradores (1994) e Christensen e colaboradores (1998) explicam o comprometimento de memória episódica já nos estágios iniciais da DA como uma falha na aquisição inicial ou codificação de informações novas. O declínio de memória episódica foi, portanto, um marcador neuropsicológico dos estágios iniciais da DA.

A associação de déficit de memória episódica e de memória de curto-prazo no grupo de pacientes com DA estudado mostrou comprometimento de múltiplos sistemas de memória e ausência de dissociação entre sistema de memória de longo-prazo e curto-prazo. Assim, o grupo de pacientes com DA apresentou alterações neuropsicológicas sugestivas de disfunção em estruturas do lobo temporal e frontal diferindo dos pacientes amnésicos e dos casos pré-clínicos que apresentam exclusivamente déficit de memória episódica (Almkvist, 1996; Squire, 1986).

\section{Assimetria entre Memória Verbal e Visual}

A assimetria entre aprendizagem de estímulos verbais e visuais intragrupo foi avaliada para verificar a existência de comprometimento mais acentuado de um dos hemisférios cerebrais. Segundo Lezak (1995), memória verbal avalia o funcionamento do lobo temporal dominante (esquerdo para destros) e a memória visual avalia o lobo temporal não dominante (direito para destros). Os pacientes com DA apresentaram comprometimento da memória verbal e visual. O grupo controle apresentou melhor desempenho no teste verbal, o que não foi observado no grupo dos pacientes com DA. Ao contrário, os pacientes apresentaram comprometimento acentuado na tarefa verbal semelhante ao da tarefa visual. Desta forma, foi possível levantar a hipótese de que a DA promove um maior declínio da memória verbal quando comparada à visual.

Os achados da comparação entre os grupos reforçaram esta hipótese. Entre os testes de memória episódica, o teste Palavras (memória verbal) foi o que melhor discriminou o grupo com DA do grupo controle. Além disso, o teste Palavras foi o que apresentou maior área sob a curva ROC, a sua variável de porcentagem de acertos classificou corretamente $96 \%$ dos sujeitos e sua variável de tempo de reação, 93\%. Estes achados neuropsicológicos indicam um comprometimento bilateral dos lobos temporais, com predomínio do comprometimento funcional do lobo temporal esquerdo (dominante).

\section{Tempo de Reação de Escolha}

O grupo dos pacientes com DA apresentou tempo de reação aumentado em todos os testes de tempo de reação de escolha quando comparado com o grupo controle, indicando que a DA promoveu lentificação do processamento das informações. Esta lentificação cognitiva evidenciada no grupo com DA envolveu estruturas neurais perceptivas, motoras e associativas. $\mathrm{O}$ tempo de reação pode ser considerado, portanto, uma medida indireta da capacidade funcional do sistema nervoso central. Os resultados evidenciados concordaram com os estudos de Kerr e colaboradores (1992) e Nebes e Brady (1992) que, utilizando procedimentos diferentes, observaram aumento do tempo de reação em pacientes nos estágios iniciais da DA.

Além disso, o aumento significativo do tempo de reação médio do grupo com $\mathrm{DA}$ em relação ao grupo controle em todas as tarefas reforçou a hipótese descrita por Nebes e Brady (1992) da linearidade e generalidade funcional do tempo de reação. Segundo os autores, a DA gera uma lentificação generalizada dos processos cognitivos que é essencialmente independente da natureza da tarefa e das funções cognitivas envolvidas.

Neste contexto, foi possível levantar a hipótese de que o tempo de reação seria subjacente a todas as funções cognitivas, pois representaria a velocidade com que as informações são processadas pelo sistema nervoso central independente da acurácia deste processamento. Em relação ao número de acertos, os pacientes com DA apresentaram porcentagem de acertos diminuída apenas no teste Formas com Memória de Curto-Prazo. Este resultado mostrou que apesar de os pacientes apresentarem uma lentificação no processamento, não mostraram déficit de percepção visual. O comprometimento foi evidenciado apenas na tarefa que demandava, além de percepção visual, memória de curtoprazo.

Os resultados da análise das curvas ROC de cada variável reforçaram os achados acima abordados. Todas as variáveis de tempo de reação classificaram corretamente $80 \%$ ou mais dos sujeitos, apresentando alta capacidade para diferenciar o grupo dos pacientes com DA do grupo controle. Em contraste, as variáveis que mediram porcentagem de acertos dos testes de tempo de reação de escolha, com exceção do que incluiu a avaliação da memória de curto-prazo, não apresentaram capacidade discriminativa.

Mesmo nos testes de memória episódica, a mensuração do tempo de reação como variável dependente foi altamente sensível e específica, mostrando que a associação do tempo de reação com a porcentagem de acertos poderia proporcionar uma análise mais refinada do grau de comprometimento de memória que o número de acertos exclusivamente.

Psicologia: Reflexão e Crítica, 2001, 14(2), pp. 305-316 


\section{Memória de Curto-Prazo}

Ao comparar a diferença entre o desempenho intragrupo no teste Formas Diretas com o do teste Formas com Memória de Curto-Prazo foi possível verificar o efeito da inclusão de um intervalo curto de tempo entre os estímulos no tempo de reação médio e na porcentagem de acertos. O grupo com DA e o grupo controle apresentaram porcentagem de acertos significativamente maior no teste Formas Diretas comparado ao teste Formas com Memória de CurtoPrazo. A magnitude da diferença entre os testes foi maior para o grupo com DA, mostrando que apesar dos idosos normais diminuírem o seu desempenho com a introdução de um intervalo de tempo entre os estímulos, os pacientes com DA apresentaram um declínio significativamente maior.

Em relação ao tempo de reação, enquanto os pacientes com DA apresentaram um tempo de reação aumentado no teste Formas com Memória de CurtoPrazo quando comparado com o teste Formas Diretas, este aumento não foi observado no grupo controle. A diminuição do número de acertos e um aumento no tempo de processamento das informações no grupo com DA indicaram um comprometimento da memória de curto-prazo nos estágios iniciais da doença.

Este déficit de memória de curto-prazo pode ser explicado por uma falha no controle atencional do centro executivo, um dos sub-componentes do modelo de memória operacional descrito por Baddeley e Hitch (1974). O centro executivo tem como função selecionar os estímulos relevantes, coordenar e manipular estímulos de naturezas e fontes diversas. Uma alteração no funcionamento deste centro executivo na DA tem sido descrita como explicação para o baixo desempenho dos pacientes em diversas tarefas cognitivas (Becker, 1988; Morris \& Baddeley, 1988). Baddeley e colaboradores (1986, 1991), ao proporem modelos experimentais para testar esta hipótese explicativa, pressupuseram que qualquer procedimento que aumentasse a demanda ou a complexidade da tarefa causaria uma redução no desempenho dos pacientes com DA. Neste sentido, o baixo desempenho dos pacientes com DA no teste Formas com Memória de Curto-Prazo poderia estar representando uma falha no controle atencional do centro executivo da memória operacional. Goldman-Rakic (1992) descreveu, utilizando métodos funcionais de neuroimagem, um envolvimento do córtex pré-frontal em tarefas usadas como modelo de memória operacional. Baddeley (1996) reforçou esta idéia afirmando que pacientes com lesões no lobo frontal apresentaram comprometimento de memória operacional associado a déficit de funções executivas.

\section{Conclusões}

As principais características neuropsicológicas dos estágios iniciais da DA foram: a) tempo de reação aumentado indicando uma lentificação do processamento das informações pelo sistema nervosos central; b) comprometimento da memória episódica observado em todos os testes, mais acentuado para o teste de memória verbal, indicando um acometimento bilateral dos lobos temporais, com predomínio do hemisfério dominante (esquerdo); c) comprometimento da memória de curtoprazo que tem sido correlacionada com disfunção no lobo frontal.

A associação da mensuração do tempo de reação e tipo de resposta nos testes de memória episódica proporcionaram uma análise mais refinada do desempenho dos pacientes com DA. Como a memória episódica encontrou-se muito comprometida (em alguns testes atingindo efeito "solo"), a mensuração do TR poderia ser um procedimento útil para monitorar a evolução da doença.

\section{Referências}

Almkvist, O. (1996). Neuropsychological features of early Alzheimer's disease: Preclinical and clinical stages. Acta Neurologica Scandinavica Supplement, 165, 63-71.

Amaducci, L. A. \& Lippi, A. (1990). Descriptive and analytic epidemiology of Alzheimer's disease. Em K. Maurer, P. Riederer \& H. Beckmann. (Orgs.), Alzheimer's disease. Epidemiology, neuropathology, neurochemistry, and clinics (pp. 41-50). Viena: Springer-Verlag.

Bachman, D. L., Wolf, P. A., Linn, R. T., Knoefel, J. E., Cobb, J. L., Belanger, A. J., White, L. R. \& D'Agostino, R. B. (1993). Incidence of dementia and probable Alzheimer's disease in a general population: The Framingham study. Neurology, 43, 515-519.

Baddeley, A. D. \& Hitch, G. J. (1974). Working memory. Em G. Bower (Org.), Recent advances in learning and motivation (pp. 47-90). Londres: Academic Press.

Baddeley, A. D., Logie, R., Bressi, S., Della Sala, S. \& Spinnler, H. (1986). Dementia and working memory. Quarterly Journal of Experimental Psychology, 38, 603-618.

Baddeley, A. D., Bressi, S., Della Sala, S., Logie, R. \& Spinnler, H. (1991). The decline of working memory in Alzheimer's disease: longitudinal study. Brain, 114, 2521-2543.

Baddeley, A. D. (1996). The fractionation of working memory. Proceedings of National Academy of Science of USA, 93, 13468-13472.

Becker, J. T. (1988). Working memory and secondary memory deficits in Alzheimer's disease. Journal of Clinical and Experimental Neuropsychology, 10,739-753.

Caramelli, P., Robitaille, Y., Laroche-Cholette, A., Nitrini, R., Gauvreau, D., Joanette, Y. \& Lecours, A. R. (1998). Structural correlates of cognitive deficits in a selected group of patients with Alzheimer's disease. Neuropsychiatry, Neuropsychology, and Behavioral Neurology, 11, 184-190. 
Charchat, H (1999). Desenvolvimento de uma bateria de testes neuropsicológicos computadorizados para o diagnóstico precoce da Doença de Alz̧heimer. Dissertação de Mestrado não-publicada. Curso de Pós-Graduação de Neurociências e Comportamento, Instituto de Psicologia, Universidade de São Paulo. São Paulo, SP.

Christensen, H., Kopelman, M.D., Stanhope, N., Lorentz, L. \& Owen, P. (1998). Rates of forgetting in Alzheimer dementia. Neuropsychologia, 36, 547-557.

Fratiglioni, L., Grut, M., Forsell, Y., Viitanen, M., Grafström, M. \& Holmen, K. (1991). Prevalence of Alzheimer disease and other dementias in an elderly urban population: relationship with age, sex, and education. Neurology, 41, 1886-1892.

Goldman-Rakic, P. S. (1992). Working memory and the mind. Scientific American, 267, 111-117.

Greene, J. D. \& Hodges, J. R. (1996). The fractionation of remote memory. Evidence from a longitudinal study of dementia of Alzheimer type. Brain, 119, 129-142.

Häfner, H. (1990). Epidemiology of Alzheimer's disease. Em K. Maurer, P. Riederer \& H. Beckmann. (Orgs.), Alzheimer's Disease. Epidemiology, neuropathology, neurochemistry, and clinics (pp. 23-40). Viena: Springer-Verlag.

Herrera, E., Caramelli, P. \& Nitrini, R. (1998). Estudo epidemiológico populacional de demência na cidade de Catanduva - estado de São Paulo - Brasil. Revista de Psiquiatria Clínica, 25, 70-73.

Hodges, J. R. \& Patterson, K. (1995). Is semantic memory consistently impaired early in the course of Alzheimer's disease? Neuroanatomical and diagnostic implications. Neuropsychologia, 33, 441-459.

Hughes, C. P., Berg, L., Danzinger, W.L., Coben, L. A. \& Martin, R. L. (1982). A new clinical scale for the staging of dementia. British Journal of Psychiatry, 140, 566-572.

IBGE - Instituto Brasileiro de Geografia e Estatística (1994). Projeção preliminar da população do Brasil para o período de 1980-2020. Rio de Janeiro: IBGE, Diretoria de Pesquisas.

Kerr, B., Calogero, M., Vitiello, M. V., Prinz, P. N., Williams, D. E. \& Wildie, F. (1992). Letter matching: Effects of age, Alzheimer's disease, and major depression. Journal of Clinical and Experimental Neuropsychology, $14,478-498$.
Lezak, M. (1995). Neuropsychological assessment. Nova York: Oxford University Press.

McKhann, G. D., Drachman, D., Folstein, M., Katzman, R., Price, D. \& Stadlan, E. M. (1984). Clinical diagnosis of Alzheimer's disease: Report of the NINCDS-ADRDA work group under the auspices of the department of health and human services task force on Alzheimer's disease. Neurology, 34, 939-944.

Mitrushina, M., Satz, P., Drebing, C., Van Gorp, W., Mathews, A., Harker, J. \& Chervinsky, A. (1994). The differential pattern of memory deficit in normal aging and dementias of different etiology. Journal of Clinical Psychology, 50, 246-252.

Morris, R. G. \& Baddeley, A. (1988). Primary and working memory functioning in Alzheimer-type dementia. Journal of Clinical and Experimental Neuropsychology, 10, 279-296.

Müller, G., Richter, R. A., Weisbrod, S. \& Klingberg, F. (1991). Reaction time prolongation in the early stage of presenile onset Alzheimer's disease. European Archives of Psychiatric and Clinical Neuroscience, 241, 4648.

Nebes, R. D. \& Brady, C. B. (1992). Generalized cognitive slowing and severity of dementia in Alzheimer's disease: Implication for interpretation of response-time data. Journal of Clinical and Experimental Neuropsychology, 14, 317-326.

Schneider, W. (1995). Mel Professional User's Guide. Pittsburgh: Psychology Software Tools.

Squire, L. (1986). Mechanisms of memory. Science, 232, 1612-1619.

Tulving, E. (1995). Organization of memory: Quo vadis? Em S. Gazzaniga (Org.), The cognitive neurosciences (pp. 839-847). Massachusetts: MIT.

United Nations. (1986). Report of the interregional seminar to promote the implementation of the international plan of action on aging. United Nations Publications and Sales, 5, 86.

\section{Sobre os autores:}

Helenice Charchat Fichman é Mestre e Doutoranda em Psicologia pelo Instituto de Psicologia, área de concentração neurociências e comportamento da Universidade de São Paulo. Neuropsicóloga e pesquisadora do Grupo de Neurologia Cognitiva e do Comportamento da Clínica Neurológica do Hospital das Clínicas da Universidade de São Paulo. Pesquisadora do Laboratório de Neurociências Cognitivas e Processamento de Informação Neural. Professora da disciplina de Psicologia Experimental da Universidade Estácio de Sá.

Ricardo Nitrini é Doutor em Medicina pelo Departamento de Neurologia da Faculdade de Medicina da Universidade de São Paulo Professor associado do Departamento de Neurologia da Faculdade de Medicina da Universidade de São Paulo Coordenador do Curso de Pós-Graduação do Departamento de Neurologia da Faculdade de Medicina da Universidade de São Paulo. Chefe do grupo de Neurologia Cognitiva e do Comportamento da Clínica Neurológica do Hospital das Clínicas da Faculdade de Medicina da Universidade de São Paulo.

Paulo Caramelli é Doutor em Medicina pelo Departamento de Neurologia da Faculdade de Medicina da Universidade de São Paulo. Professor do Curso de Pós-Graduação em Neurologia da Faculdade de Medicina da Universidade de São Paulo. Médico Assistente da Divisão de Clínica Neurológica do Hospital das Clínicas da Faculdade de Medicina da Universidade de São Paulo. Pesquisador do Grupo de Neurologia Cognitiva e do Comportamento da Clínica Neurológica do Hospital das Clínicas da Faculdade de Medicina da Universidade de São Paulo.

Koichi Sameshima é Doutor em Fisiologia Humana pelo Instituto de Ciências Biomédicas da Universidade de São Paulo. Pós-Doutorado na University of California San Francisco entre 1992 e 1994. Professor da Disciplina de Informática Médica do Departamento de Patologia da Faculdade de Medicina da Universidade de São Paulo. Pesquisador do Laboratório de Investigação Médica do Departamento de Neurologia da Faculdade de Medicina da Universidade de São Paulo. Coordenador do grupo de pesquisa denominado Laboratório de Neurociências Cognitivas e Processamento de Informação Neural. 